\title{
Application of Smart Phone Calculations for Commodity Delivery Truck Drivers
}

\author{
Ming-Kuei Yeh \\ Department of Information Management \\ National Taipei University of Business \\ Taipei City, Republic of China \\ e-mail: c8880@ms21.hinet.net, jamesyeh@ntub.edu.tw
}

\author{
Zih-Ping Ho \\ Business Intelligence Research Center \\ National Taipei University of Business \\ Taipei City, Republic of China \\ e-mail: tp.her@msa.hinet.net, zihping@ntub.edu.tw
}

\begin{abstract}
The Internet of things (IOT), real-time positioning, body sensor monitoring, and human decision support systems have become hot topics over the last two years. Commodity delivery truck drivers face these heavy tasks directly and have also become a key to success of the new IOT trend. A commodity delivery truck driver does not have sufficient time to make decisions when on the road. He (or she) has to know the location of nearby gas stations, fast food restaurants, toilets, temporary rest places, waste bottle recycling, customer shops, and mainly product distribution missions. This study focuses on a smart phone applying remote server as intelligent system. This intelligent system can deal with product distribution sub problem, waste bottle recycling routes sub problem, on-line real time Annealing heuristics (SAH) calculation, finding support shops for a driver and body sensor monitor; all integrating on a smart phone display. In previous research, there is only a limited amount of literature available on routes planning hybrid driver's body real time monitoring and giving suggestion. This study establishes a pioneer research about optimization product distribution and human behavior interacting intelligent system, sensors also embedded into this system. Through validations, the study offered fundamental data for optimization of this problem and useful calculations for a commodity delivery driver. Future research suggests comparing more heuristics results to these SAH results, or build more constraints to help a driver to face his (or her) daily multiple routes planning choices.
\end{abstract}

Keywords- positioning applications; Simulated Annealing Heuristics; SAH; mobile computing; routes and map planning; business intelligence system

\section{INTRODUCTION}

The Internet of things (IOT), real-time positioning, body sensor monitoring, and human decision support systems have become a hot topic over the last two years. Commodity delivery truck drivers face these heavy tasks directly and have also become a key to success of the new IOT trend. A commodity delivery truck driver does not have sufficient time to make decisions when on the road. He (or she) has to know the location of nearby gas stations, fast food restaurants, toilets, temporary rest places, waste bottle recycling, customer shops, and mainly product distribution missions. This study also includes previous research [1] techniques, ECG, breathing and instant blood pressure mobile device sensors into the truck driving behavior calculations. In a real empirical case, a commodity delivery company will often add commodities by mobile phone call; the customer is not at home on a predefined time; so the driver has to change the schedule for home delivery services.

This study focuses on a smart phone applying remote server as intelligent system. This intelligent system can deal with product distribution sub problem, waste bottle recycling routes sub problem, on-line real time Annealing heuristics (SAH) calculation, finding support shops for a driver and body sensor monitor; all integrating on a smart phone display.

Our previous study [2] calculated the profit of orders from recycling products. However, this study also extended that research; but in this study we focus on integrated intelligent system application; any uncomfortable or death from overwork for a driver, the monitor system would prevent those and utilize mobile computing techniques.

The system also plots the possible solution after calculation as a figure using Google Map API, and demonstrate ECG, breathing and instant blood pressure data.

\section{LITERATURE REVIEW}

A geographical related intelligent system has become popular due to the $4 \mathrm{G}$ techniques of the recent intelligent phone trend. Products, such as Google releasing Google Maps API, would make it possible to see any local map on earth; furthermore, proceed with more value-added applications. The previous literatures which were related to this study were as follows.

Southworth et al. [3] discussed Chicago traffic system of introducing preferential truck routing and terminals clustering are simulated. By channelizing heavy truck flows along a subset of the region's major highways, and linking such routes to terminal clusters, a more economical as well as environmentally appealing urban goods movement system was being sought. Their model was meant for strategic planning, and works on a partially aggregated sketch planning highway network.

Hubschneider [4] presented that many routes taken by truck drivers were suggested by navigation devices. These navigation devices did not always consider the needs of trucks. This leads to substantial problems in cities, such as trucks colliding with low bridges or taking unsuitable routes via the city center or residential areas. Hubschneider described the steps required to integrate communal routing preferences and interests into navigation systems. It showed which rules had to be considered when developing a concept for preferred routes and what the consequences were for system design. All project stages were shown, including 
implementing the preferred truck routing concept, extracting the common construction rules for modelling preference networks, consolidating the findings from the process, and building a realistic, working navigation system. Ho [5] used a branch of dynamic programming - Dynamic order selection model to deal with operational profits of a restaurant. Ho [6] studied just-in-time orders delivery mixed outsourcing problem. Ho [2] studied geographical information management system in orders and trucks assignment problem. Ho and $\mathrm{Wu}$ [7] studied Simulated Annealing Algorithm to optimization deployment of mobile wireless base stations. In this study, the target and problem are not Ho [2][5][6][7]s' topics, but related techniques.

Gan [8] stated that a recent attempt to enhance traveler information services and alleviate congestion is by the use of graphical route information panel (GRIP). Gan proposed a travel simulator for collecting behavioral data on driver response to GRIP. The traffic network conditions and GRIP information were driven by an extended version of the validated high-order continuum model METANET, thus enhancing the realism of the hypothetical travel scenario provided by the simulator. Gan designed simulator, and a prototype simulator was successful developed. Zhou et al. [9] applied prospect theory to describe drivers' route choice behavior under variable message sign (VMS), which presented visual traffic information to assist them to make route choice decisions. Rahmani et al. [10] stated that traffic management centers take advantage of various data collection systems ranging from stationary sensors e.g. automated vehicle identification systems to mobile sensors e.g. fleet management systems. Each type of data collection system had its own advantages and disadvantages. Stationary sensors had less measurement noise than mobile sensors but their network coverage was limited. On the other hand, mobile sensors covered expand areas of road networks but they have less penetration rate and frequency of reports. In their research, the sensors were applied to do traffic management.

Babel and Zimmermann [11] investigated a route finding algorithm leading the ship's movement. It allowed to minimize the route length, the traveling time, the number of maneuvers, or other objectives. The basic idea was to embed a network in the operational area and compute a least-cost path. Instead of using a regular grid graph which strongly restricts the types of maneuvers and necessitates a path smoothing after optimization, they designed a network which was especially tailored to the maneuverability of the vessel. Each path in this network represented a continuous-curvature track based on combinations of clothoids and straight line segments. The approach allowed a large variety of maneuvers, hence high-quality solutions were achievable provided a sufficiently dense network. They applied dense network concept to guide the routes. Zhang and Duan [12] formulated the global route planning problem for the unmanned aerial vehicles (UAVs) as a constrained optimization problem in the three-dimensional environment and proposed an improved constrained differential evolution (DE) algorithm to generate an optimal feasible route. The flight route was designed to have a short length and a low flight altitude. The multiple constraints based on the realistic scenarios were taken into account, including maximum turning angle, maximum climbing or gliding slope, terrain, forbidden flying areas, map and threat area constraints. The proposed DE-based route planning algorithm combined the standard DE with the level comparison method and an improved strategy was proposed to control the satisfactory level. To show the high performance of the proposed method, they compare the proposed algorithm with six existing constrained optimization algorithms and five penalty function based methods. Numerical experiments in two test cases were carried out. They proposed algorithm demonstrated a good performance in terms of the solution quality, robustness, and the constraint-handling ability. Our study is truck routes, not a flight routes; however, the system still has to calculate in a very short time and guide the right routes direction.

Although several literatures were related to routes planning, there is only a limited amount of literature available on routes planning hybrid driver's body real time monitoring and giving suggestion.

\section{PROBLEM FORMULATION}

This research adapts finding the minimal penalty of product distribution and waste bottle recycling, $C_{i j}$, as the model. A dynamic selection model [2][5], previous research, helps this study construct a more precise problem formulation. In this study, let $N$ denote set of nodes. Let $n$ denote number of nodes. Let $i, j$ denote node number, $i \neq j$. Let $c t(i, j)$ denote cost function from transportation node $i$ to node $j$. Let $c e d(i, j)$ denote ED penalty cost from transportation node $i$ to node $j$, it's also an Early and Delay job optimization sub problem. Let $c w(i, j)$ denote waste penalty cost from transportation node $i$ to node $j$, it means carriage waste bottle cost and storage cost of waste bottle on the customer side. Let $\operatorname{live}(i, j)$ denote live penalty cost from transportation node $i$ to node $j$. Let $C_{i j}$ denote penalty function from node $i$ to node $j$. Let $x_{i j}$ denote a binary decision variable. The model is shown as follows.

$N$ : set of nodes

$n$ : number of nodes

$i, j$ : node number, $i \neq j$

$c t(i, j)$ : cost from transportation node $i$ to node $j$

ced $(i, j)$ : ED penalty cost from transportation node $i$ to node $j$, it's also an Early and Delay job optimization sub problem.

$c w(i, j)$ : waste penalty cost from transportation node $i$ to node $j$, it means carriage waste bottle cost and storage cost of waste bottle on the customer side. 


$$
\begin{aligned}
& \text { live }(i, j) \text { : live penalty cost from transportation node } i \text { to } \\
& \text { node } j \text {, it means if causing fatigue or physical } \\
& \text { discomfort to a driver. } \\
& \text { In this study, it calculated physiological needs } \\
& \text { to eat, to a toilet, to take a rest and death from } \\
& \text { overwork. } \\
& C_{i j} \text { : penalty function from node } i \text { to node } j \\
& c_{i j}=f(i, j)=c t(i, j)+\operatorname{ced}(i, j)+c w(i, j)+\operatorname{live}(i, j) \\
& X_{i j}=\left\{\begin{array}{ll}
1, & \text { the path goes from node } i \text { to node } j \\
0, & \text { otherwise }
\end{array}\right\}
\end{aligned}
$$

The MINLP (Mixed integer non-linear programming) model to minimize the penalty cost (1) is as follows:

\section{Min}

$$
\sum_{i \in N} \sum_{j \in N} c_{i j} x_{i j}
$$

s.t.

$$
\sum_{i=0}^{n} x_{i j}=1
$$

$$
\forall
$$$$
\text { j }
$$

$$
\sum_{j=0}^{n} x_{i j}=1
$$$$
\forall
$$$$
\text { i }
$$$$
x_{i j} \in\{0,1\} \quad \forall
$$$$
i, j
$$

In (2) and (3), each node should visit at one time. Equation (4) declares artificial binary decision variable constraints.

\section{IMPROVED SimUlated ANNEALING ALgORITHM}

The SA Algorithm for this problem is as follows:

The decision variables is $X_{i j}$.

Step 1: Initialization

Randomize an initial solution

The solution represents the path flow in the whole system.

Step 2: Objective function evaluation

Evaluate the objective function for the initial

solution

Step 3: Neighbour solution generation

Based on previous solution to generate a new

solution
Step 4: Objective function evaluation

Evaluate the objective function for the neighbour solution

Step 5: Comparison

If the new solution is better than the original solution, then replace it. Otherwise, use the Boltzman distribution [7] for determining whether to accept the new solution or reject dependent on relative magnitude of $\Delta \mathrm{E}$ and $T$ values.

Step 6: Cooling

In each temperature, the procedure generates a fixed number of neighbour solutions, then $\alpha$ would reduce the temperature of the annealing process.

Step 7: Stopping

Has iteration reached a pre-defined number? If yes, then Stop the searching process. Otherwise, the procedure goes back to step 3 .

In this study, step 3 and 5 were improved and extended, the software let MP denote the memory of the previous (5) path sets.

$$
x_{i j} \rightarrow \mathrm{MP}
$$

Besides, the neighbour solution generation keeps the top 10 paths as a candidate neighbour. In each iteration,it randomly selects one of these ten candidate neighbours as a previous solution in Step 3.

This study also revised Step 1, the initial solution is setting geographical constraints. The nodes which belong to the same distinct must be preferentially allocated. It would accelerate the searching efficiency in the initial step.

\section{COMPUTER RESULTS AND DISCUSSION}

In this section, the algorithm was coded in Visual Basic computer language with Celeron (R) Dual-Core CPU at 2.10 $\mathrm{GHz}$ and $1 \mathrm{~GB}$ of RAM memory. This MINLP model is a strong NP-hard problem [13]. Since the proposed model is a new problem, no published solution method is available for comparison. Thus, this research conducted an experimental design, at different nodes, for comparing the performance of heuristics. The parameters of SA were listed in Table 1. The research results also showed in Figure 1.

The smart phone used PHP computer language to launch an exe file coded by Visual Basic, and resubmitted the necessary data into Google Map API. The process required 0.9 seconds. The Google Map API techniques were based on textbooks [14]. The dynamic plot support shops for a truck driver were shown as the following two pictures. Figure 2 was nearby gas stations. Figure 3 was nearby restaurants. Figure 4 showed body sensor data of ECG, breathing and 
instant blood pressure from mobile device sensors. In figure 4, the smart phone only reminded the truck driver of his body's current condition and recorded the results. The smart phone algorithm was not designed to restrict his long term driving behaviors. But if the truck driver needs to sleep, the breathing sensor would alarm him that he in danger, and therefore must take a rest instantly. Also if instant blood pressure data is within the dangerous zone, the sensor would alarm the driver to take a rest instantly for the prevention of a stroke.

TABLE I. SET OF PARAMETERS FOR SA

\begin{tabular}{|l|l|}
\hline Parameter & Value \\
\hline Cooling Rate $\alpha$ & 0.90 \\
\hline$T_{0}$ & $100^{\circ} \mathrm{C}$ \\
\hline$T_{n}$ & $0^{\circ} \mathrm{C}$ \\
\hline Iterations at the $T_{i}$ & 10 \\
\hline Threshold Rate to $\mathrm{P}(\Delta \mathrm{E})$ & 0.50 \\
\hline Tabu & 0 \\
\hline [1] Termination time (sec) & 10 \\
\hline
\end{tabular}

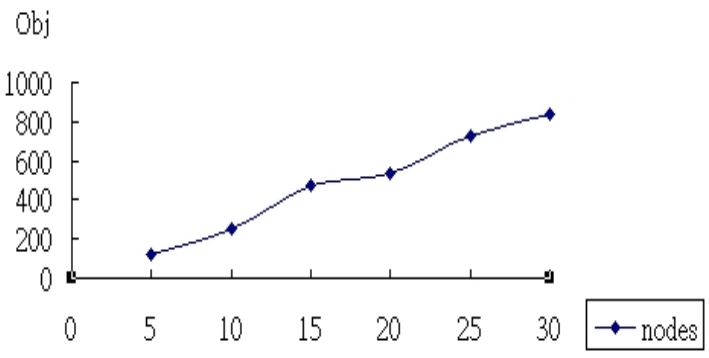

Figure 1. Comparison SAH results at different nodes.

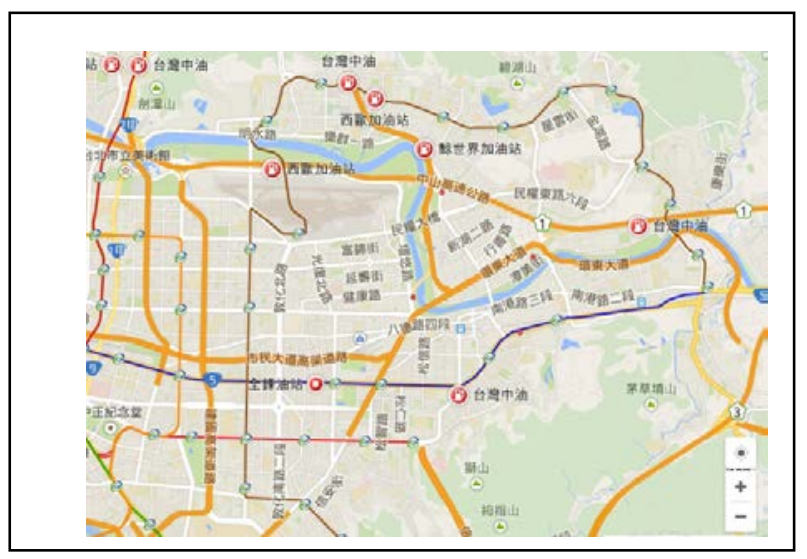

Figure 2. Nearby gas stations.

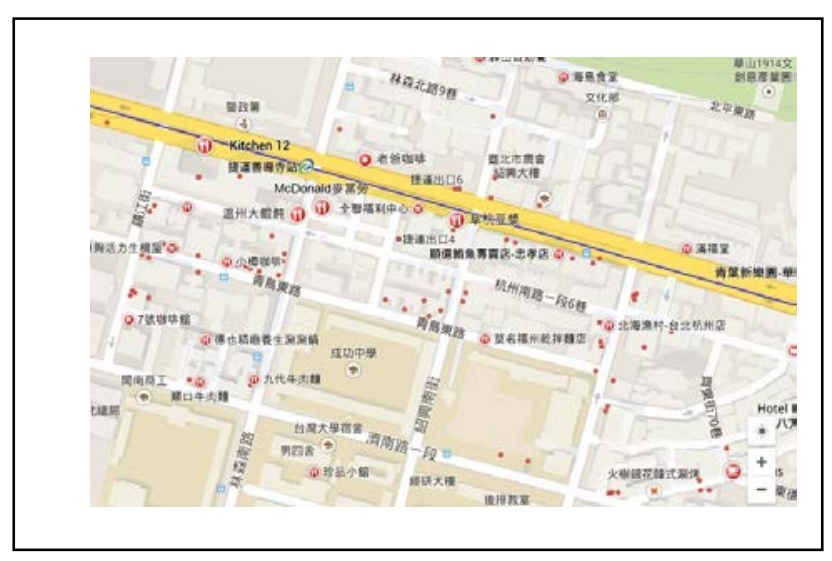

Figure 3. Nearby restaurants.

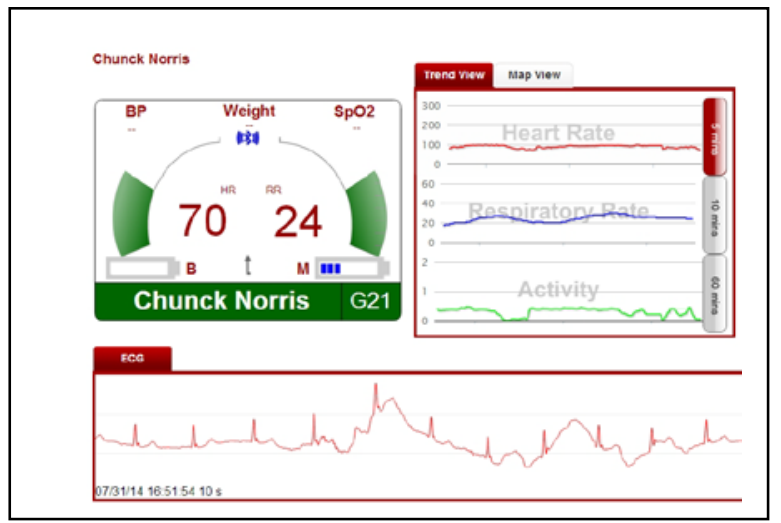

Figure 4. Body sensor data collected from mobile device.

\section{CONCLUSIONS}

In previous research, there is only a limited amount of literature available on routes planning hybrid driver's body real time monitoring and giving suggestion. This study establishes a pioneer research about optimization product distribution and human behavior interacting intelligent system, sensors also embedded into this system. Through validations, the study offered fundamental data for optimization of this problem and useful calculations for a commodity delivery driver.

Future research suggests comparing more heuristics results to these SAH results, or build more constraints to help a driver to face his (or her) daily multiple routes planning choices.

\section{ACKNOWLEDGMENT}

The work is supported by the project of Ministry of Science and Technology of Republic of China. The grand number is MOST 103-2221-E-141-005. 


\section{REFERENCES}

[1] M. K. Yeh and Z. P. Ho, "Health information reminder- Elderly people living in cloud computing architecture as an example," Energy Edu. Sci. and Tech.-Part A. Energy Sci. and Res., vol. 32, 2014, pp. 5771-5776.

[2] Z. P. Ho, "Geographical information of dynamic carbon footprints management system in orders and trucks assignment problem," Advanced Materials Res. J., vol. 518-523, 2012, pp. 5611-5615.

[3] F. Southworth, Y. J. Lee , and D. Zavattero, "A system model of primary truck route designation and terminals clustering," Transportation Res. Part A: General, vol. 20, 1986, pp. 351-360.

[4] M. Hubschneider, "Preferred truck routes meet navigation," Procedia Social and Behavi. Sci., vol. 39, 2012, pp. 490-494.

[5] Z. P. Ho, "An application of new approach for dynamic orders selection to maximize restaurant operational profits," Adv. Mat. Res. J., vol. 472-475, 2012, pp. 380-385.

[6] Z. P. Ho, "Just-in-time orders delivery mixed outsourcing problem," App. Mechanics and Mat., vol. 220-223, 2012, pp. 246-250.

[7] Z. P. Ho and C. S. Wu, "Application of Simulated Annealing Algorithm to optimization deployment of mobile wireless base stations," Adv. in Intelligent and Soft Compu., vol. 169, 2012, pp. 665-670.
[8] H. C. Gan, "A travel simulator for collecting data on driver response to graphical route information panel," Procedia - Soc. and Behavi. Sci., vol. 96, 2013, pp. 1091-1100.

[9] L. Zhou, S. Zhong, S. Ma , and N. Jia, "Prospect theory based estimation of drivers risk attitudes in route choice behaviors," Accident Analysis \& Prevention, vol. 73, 2014, pp. 1-11.

[10] M. Rahmani, E. Jenelius , and H. N. Koutsopoulos, "Floating car and camera data fusion for non-parametric route travel time estimation," Procedia Compu. Sci., vol. 37, 2014, pp. 390-395.

[11] L. Babel and T. Zimmermann, "Planning safe navigation routes through mined waters,” Euro. J. of Operational Res., vol. 241, 2015, pp. 99-108.

[12] Xiangyin Zhang and Haibin Duan, "An improved constrained differential evolution algorithm for unmanned aerial vehicle global route planning,” App. Soft Compu., vol. 26, 2015, pp. 270-284.

[13] K. H. Rosen, "Discrete mathematics and its applications," McGraw Hill press, 2006.

[14] C. Jiang and X. P. Gong, Google API solution and developmentCombination of Google Map and Google Earth, 2nd ed., Taipei City: Sung Gang Asset Management Corp. Limited, 2011. 